\title{
Reconhecimento de fala no nível de máximo conforto em pacientes adultos com perda auditiva neurossensorial
}

\author{
Speech recognition in the maximum comfort level in adults \\ with sensorineural hearing loss
}

\author{
Zuleica Costa Zaboni' ${ }^{1}$, Maria Cecília Martinelli Iorioº
}

\begin{abstract}
RESUMO
Objetivo: Pesquisar o Índice Percentual de Reconhecimento de Fala (IPRF) no nível de máximo de conforto em adultos com perda auditiva neurossensorial de grau leve a moderadamente severo até $60 \mathrm{~dB}$ NA. Métodos: Os indivíduos avaliados foram agrupados de acordo com o grau de perda auditiva (Grupos I, II e III). Feito isso, os grupos foram subdivididos (IA, IB, IIA, IIB, IIIA, IIIB) conforme explicado a seguir. No subgrupo A de cada um dos grupos, foi determinado o IPRF a $40 \mathrm{~dB}$ NS, obtendo-se as respostas iniciando-se pela orelha direita. O paciente foi solicitado a informar quão confortável estava o som neste nível de apresentação dos estímulos, utilizando-se para obter a informação, uma escala com quatro descritores: baixo, confortável, alto e alto demais. Depois disso, foi pesquisado o nível de máximo conforto (NMC) e nessa intensidade foi obtido o IPRF. No subgrupo B de cada um dos grupos, foi realizado o mesmo procedimento, porém obtendo-se primeiramente o IPRF no NMC, e a seguir a $40 \mathrm{~dB}$ NS. Nesse subgrupo, o teste foi iniciado na orelha esquerda. Resultados: Após a avaliação dos indivíduos dos três grupos foi calculado o nível de audição médio em que os indivíduos referiram maior conforto que variou de 25 a 32,95 dB NS. No NMC de apresentação dos estímulos, houve maior percentagem de acertos de palavras. Conclusão: A avaliação do IPRF no nível de máximo conforto para indivíduos com perda auditiva neurossensorial de grau leve a moderadamente severo, proporciona melhores resultados de Reconhecimento de Fala.
\end{abstract}

Descritores: Perda auditiva neurosensorial; Limiar auditivo; Audiometria da fala; Percepção da fala

\section{INTRODUÇÃO}

A comunicação é a base para a interação social dos indivíduos. Para que esta aconteça, é necessária uma boa compreensão de fala ${ }^{(1)}$.

Alguns indivíduos sentem dificuldade de comunicação em ambientes ruidosos ou queixam-se de escutar, mas não entender nesses mesmos ambientes ${ }^{(2-3)}$. Estes indivíduos podem apresentar perda auditiva neurossensorial o que pode levar a um comprometimento na inteligibilidade de fala ${ }^{(4)}$.

Sabe-se que as perdas auditivas neurossensoriais possuem múltiplas causas e, que, na sua maioria, não são passíveis de tratamento médico ou cirúrgico. A intervenção possível nesses casos é a (re) habilitação por meio da adaptação de próteses

Trabalho realizado no Departamento de Fonoaudiologia da Universidade Federal de São Paulo - UNIFESP - São Paulo (SP), Brasil.

(1) Especializanda em Distúrbios da Comunicação Humana pelo Departamento de Fonoaudiologia da Universidade Federal de São Paulo - UNIFESP - São Paulo (SP), Brasil.

(2) Livre-docente, Professora associada do Departamento de Fonoaudiologia da Universidade Federal de São Paulo - UNIFESP - São Paulo (SP), Brasil. Endereço para correspondência: Zuleica Costa Zaboni. R. Alba, 2088/95, Vila Santa Catarina, São Paulo (SP), Brasil, CEP: 04346-000. E-mail: zuleicafono@yahoo.com.br

Recebido em: 20/8/2008; Aceito em: 20/7/2009 auditivas. Os testes de fala constituem indicadores importantes sobre o prognóstico do processo de seleção e adaptação de próteses auditivas. Esses testes fornecem dados sobre a eficiência comunicativa dos indivíduos, além de informações para o diagnóstico diferencial relacionadas ao local da lesão. ${ }^{(5)}$

As perdas auditivas neurossensoriais podem apresentar as seguintes características: podem surgir lentamente, agravaremse com a idade ou serem súbitas, unilaterais ou bilaterais. Quando bilaterais, o indivíduo tende a elevar o volume de sua voz. Esta pode apresentar-se distorcida nas perdas mais severas, pois o indivíduo não ouve a própria voz.

Estes pacientes podem apresentar zumbido de frequência mais alta, comparado a uma cigarra ou um apito, intermitente ou acentuado durante a noite (devido ao menor barulho), dificultando o sono ${ }^{(6)}$, levando inclusive ao isolamento, distúrbios de personalidade, irritabilidade, capacidade de atenção reduzida e vivacidade diminuída. ${ }^{(7)}$

O reconhecimento de fala é afetado na maioria dos casos ${ }^{(8)}$ pelo comprometimento das células sensoriais da orelha interna, sendo pior quanto maior a perda auditiva. Se as frequências da fala $(500 \mathrm{~Hz}, 1000 \mathrm{~Hz}$ e $2000 \mathrm{~Hz})$ estiverem menos afetadas, o reconhecimento de fala pode estar menos comprometido.

Quando a lesão é sensorial pode acontecer o fenômeno do recrutamento, no qual o paciente apresenta alteração no 
crescimento da sensação de intensidade ${ }^{(9)}$, de tal forma que um pequeno aumento na intensidade representa uma elevação desproporcional na sensação dos sons. Assim sendo, o recrutamento modifica o padrão da curva logoaudiométrica. Na lesão neural, o reconhecimento de fala é ruim, não compatível com os limiares audiométricos e a intervenção é distinta da que acontece nas perdas sensoriais. O reconhecimento de fala é, portanto, uma medida muito importante no processo de (re) habilitação do deficiente auditivo; a avaliação do reconhecimento de fala revela como o indivíduo responde a estímulos de fala em condições de escuta satisfatórias.

A compreensão da fala é um dos requisitos fundamentais para a eficiência da comunicação; portanto, a avaliação da capacidade auditiva de um indivíduo não pode ser restrita apenas à sua habilidade de captar tons puros - como na audiometria tonal - o reconhecimento de fala nem sempre pode ser presumido pelos limiares tonais, o que torna a logoaudiometria um instrumento clínico indispensável ${ }^{(10)}$. A habilidade de compreender a fala pode ser investigada por meio do teste $\mathrm{IPRF}^{(11)}$ (Índice Percentual de Reconhecimento de Fala). O IPRF é um teste supraliminar e seus resultados são expressos em porcentagem de acertos no reconhecimento das palavras.

É recomendado o uso de listas monossilábicas foneticamente balanceadas para a realização do teste. Alguns autores ${ }^{(12)}$ defendem que, mais importante que o balanceamento fonético, é a familiaridade e o significado dos vocábulos para o paciente. Na prática clínica são utilizados monossílabos com base na ocorrência dos fonemas da língua portuguesa. São utilizadas listas de vocábulos monossilábicos e, quando o reconhecimento de fala é ruim pode-se utilizar listas de vocábulos dissílabos e trissílabos, pois quanto maior a extensão de um vocábulo mais fácil é o reconhecimento do mesmo.

Dessa forma, o objetivo deste estudo é pesquisar e comparar o Índice Percentual de Reconhecimento de Fala (IPRF), obtido no nível de máximo conforto (NMC) e a 40 decibel nível de sensação (dB NS) em adultos, segundo o grau de perda auditiva neurossensorial.

\section{MÉTODOS}

Este estudo foi realizado no Núcleo Integrado de Assistência Pesquisa e Ensino em Audição (NIAPEA) da Universidade Federal de São Paulo (UNIFESP). Cada indivíduo foi informado sobre o trabalho e ficou ciente dos procedimentos por meio do termo de livre consentimento, que explicava detalhadamente como o estudo evoluiria. O trabalho teve aprovação do Comitê de Ética e Pesquisa da UNIFESP protocolado sob o número 06/566.
Para a seleção dos indivíduos, os critérios de inclusão foram: idade entre 18 e 60 anos; perda auditiva neurossensorial de grau leve a moderadamente severo, com nível médio de audição (média das frequências de $500 \mathrm{~Hz}, 1000 \mathrm{~Hz}$ e 2000 $\mathrm{Hz}$ ) até $60 \mathrm{~dB}$. Foram considerados como critérios de exclusão: perda auditiva neurossensorial com componente condutivo (perda mista) e apresentar outros comprometimentos (alteração de compreensão e emissão oral).

Foram avaliados 47 indivíduos dos sexos masculino e feminino, distribuídos em três grupos conforme o grau da perda auditiva que apresentaram. O Grupo I foi composto por 17 adultos com perda auditiva neurossensorial de grau leve. $\mathrm{O}$ Grupo II, por 20 adultos com perda auditiva neurossensorial de grau moderado e o Grupo III, dez adultos com perda auditiva de grau moderadamente severo com perda de até $60 \mathrm{~dB}$ (Quadro 1). Cada um dos grupos foi subdividido em subgrupos A e B. Desta forma o subgrupo IA foi composto por nove adultos e o IB por oito, IIA por dez e o IIB por dez, IIIA por cinco e o IIIB por cinco adultos.

Inicialmente foi realizada a anamnese e a audiometria tonal liminar por via aérea e óssea, para determinação do tipo e grau da perda auditiva. As medidas desta pesquisa foram realizadas em cabina tratada acusticamente. $\mathrm{O}$ audiômetro utilizado foi da marca Interacoustics ${ }^{\circ}$ modelo AC33, com CD player acoplado.

Posteriormente, foi realizado o IPRF que foi obtido por meio de palavras gravadas. O teste pode ser realizado a viva voz sendo esse modo de apresentação o mais comumente utilizado devido à sua flexibilidade, rapidez e facilidade da aplicação ${ }^{(13)}$. Porém, a vantagem de utilizar listas de palavras gravadas é a possibilidade de padronização, assegurando sempre as mesmas condições de avaliaçãõo ${ }^{(13)}$.

O teste de reconhecimento de fala é realizado apresentando-se uma lista de 25 palavras monossilábicas (Quadro 2) em cada uma das orelhas. O material utilizado ${ }^{(14)}$ foi gravado em $\mathrm{CD}$ e constitui-se de quatro listas com 25 monossílabos (os mesmos monossílabos dispostos em quatro combinações diferentes) que são apresentadas a $40 \mathrm{~dB}$ NS, ou seja, $40 \mathrm{~dB}$ acima da média dos limiares de audibilidade de 500, 1000 e $2000 \mathrm{~Hz}$. A sequência de palavras foi apresentada de modo monoaural, de maneira que foi testada cada orelha separadamente. Estas listas foram apresentadas em dois níveis de intensidade: a 40 dB NS e no nível de máximo conforto.

As quatro listas foram agrupadas duas a duas para que cada uma delas fosse composta por 50 palavras. Desta forma, foram aplicadas alternadamente as listas que foram denominadas D1 e D2.

Quadro 1. Média dos limiares auditivos por frequência nas orelhas direita e esquerda, de acordo com o grau de perda

\begin{tabular}{|c|c|c|c|c|c|c|}
\hline \multirow[t]{2}{*}{ Frequência } & \multicolumn{2}{|c|}{ Leve (Grupo I) } & \multicolumn{2}{|c|}{ Moderado (Grupo II) } & \multicolumn{2}{|c|}{ Moderadamente severo (Grupo III) } \\
\hline & OD & OE & OD & OE & OD & OE \\
\hline $500 \mathrm{~Hz}$ & 33,75 & 36,25 & 42,85 & 48 & 60 & 60 \\
\hline $1000 \mathrm{~Hz}$ & 35 & 35,62 & 49,54 & 48,33 & 59 & 59 \\
\hline $2000 \mathrm{~Hz}$ & 39,16 & 36,6 & 49,44 & 43 & 59,83 & 60 \\
\hline $4000 \mathrm{~Hz}$ & 30 & 37 & 47,5 & 47,5 & 60 & 60 \\
\hline
\end{tabular}

Legenda: $\mathrm{OD}=$ orelha direita; $\mathrm{OE}=$ orelha esquerda 
Quadro 2. Listas para a realização do Índice de Reconhecimento de Fala

\begin{tabular}{|c|c|c|c|}
\hline \multicolumn{4}{|c|}{ Lista de palavras utilizadas para a realização do IPRF } \\
\hline \multicolumn{2}{|c|}{ D1 } & \multicolumn{2}{|c|}{ D2 } \\
\hline til & chá & dor & jaz \\
\hline jaz & dor & boi & cão \\
\hline rol & mil & til & cal \\
\hline pus & tom & rol & boi \\
\hline faz & zum & gim & nu \\
\hline gim & mel & cal & faz \\
\hline rir & til & nha & $\operatorname{gim}$ \\
\hline boi & $\operatorname{gim}$ & chá & pus \\
\hline vai & dil & tom & seis \\
\hline mel & nu & sul & nha \\
\hline nu & pus & tem & mil \\
\hline Ihe & nha & pis & tem \\
\hline cal & sul & nu & zum \\
\hline mil & jaz & cão & til \\
\hline tem & rol & vai & Ihe \\
\hline dil & tem & mel & sul \\
\hline dor & faz & rir & chá \\
\hline chá & Ihe & jaz & rol \\
\hline zum & boi & zum & mel \\
\hline nha & cal & mil & dor \\
\hline cão & rir & Ihe & vai \\
\hline tom & cão & ler & dil \\
\hline seis & ler & faz & tom \\
\hline ler & vai & seis & rir \\
\hline sul & seis & dil & ler \\
\hline
\end{tabular}

Fonte: Mangabeira PL, Pen $\mathrm{M}^{(14)}$

Legenda: IPRF = Índice Percentual de Reconhecimento de Fala

A avaliação foi realizada da seguinte maneira: para cada grupo, em metade dos indivíduos iniciou-se o teste pelo NMC e na outra metade a $40 \mathrm{~dB}$ NS. Esta estratégia foi utilizada para evitar que o aprendizado ou a familiaridade com o teste interferisse nos resultados.
No subgrupo A de cada um dos grupos, inicialmente, foi determinado o IPRF a $40 \mathrm{~dB}$ NS. O paciente informou o quão confortável estava o som neste nível de apresentação dos estímulos. Para obter a informação foi utilizada uma escala com quatro descritores: baixo, confortável, alto e alto demais. Nesse grupo, o teste foi iniciado pela orelha direita.

Foi pesquisado então, o NMC e nessa intensidade foi obtido o IPRF da mesma maneira. No subgrupo B de cada um dos grupos, foi realizado o mesmo procedimento, porém obtendo-se primeiramente o IPRF no NMC, e a seguir a 40 $\mathrm{dB}$ NS, iniciando o teste pela orelha esquerda.

Para a análise estatística, foi utilizado o teste não paramétrico de Wilcoxon para comparar as variáveis duas a duas. No teste, fixou-se em 0,05 ou $5 \%$ o nível de rejeição da hipótese de nulidade. Os resultados significantes foram destacados com asterisco $(*)$. O resultado aproximado à porcentagem de significância foi marcado com sustenido (\#).

Na complementação da análise descritiva, fizemos uso da técnica de Intervalo de Confiança para média.

\section{RESULTADOS}

Os indivíduos foram distribuídos conforme suas perdas auditivas. Para o Grupo I com perda auditiva de grau leve, a média das idades foi de 49,44 anos. Os indivíduos que compunham o Grupo II, com perda auditiva moderada, apresentaram idade média de 40 anos e o Grupo III apresentou idade média de 47,82 anos.

Inicialmente, foram investigadas as possíveis diferenças entre o IPRF obtido nas orelhas direita e esquerda no NMC e a $40 \mathrm{~dB}$ NS para os grupos I, II, III por meio do teste Wilcoxon. Esses dados foram apresentados na Tabela 1.

Na Tabela 1, são apresentados os resultados referentes ao grupo I (perda auditiva neurossensorial de grau leve). Não houve diferença estatisticamente significante entre as orelhas direita e esquerda, tanto para o IPRF no NMC quanto a 40 $\mathrm{dB}$ NS, embora os p-valores estivessem em limites marginais (tendência à significância).

Ainda na Tabela 1, observamos que nos grupos II e III que apresentavam perda auditiva neurossensorial de grau moderado e moderadamente severo, houve diferença esta-

Tabela 1. Medidas descritivas e resultado do teste Wilcoxon para comparação do Índice Percentual de Reconhecimento de Fala obtido nas orelhas direita e esquerda no NMC e a 40 dB NS nos Grupos I, II e III

\begin{tabular}{|c|c|c|c|c|c|c|c|c|c|c|c|c|}
\hline \multirow{3}{*}{$\begin{array}{l}\text { Medida } \\
\text { estatística }\end{array}$} & \multicolumn{4}{|c|}{ Leve (Grupo I) } & \multicolumn{4}{|c|}{ Moderado (Grupo II) } & \multicolumn{4}{|c|}{ Moderadamente severo (Grupo III) } \\
\hline & \multicolumn{2}{|c|}{ NMC } & \multicolumn{2}{|c|}{$40 \mathrm{~dB} N \mathrm{~S}$} & \multicolumn{2}{|c|}{ NMC } & \multicolumn{2}{|c|}{$40 \mathrm{~dB} N \mathrm{~S}$} & \multicolumn{2}{|c|}{ NMC } & \multicolumn{2}{|c|}{$40 \mathrm{~dB} N S$} \\
\hline & OD & OE & OD & OE & OD & OE & OD & OE & OD & OE & OD & OE \\
\hline Média & 98,12 & 96,71 & 97,18 & 95,53 & 96,00 & 89,90 & 93,14 & 87,62 & 92,89 & 87,11 & 83,11 & 77,78 \\
\hline Mediana & 100 & 96 & 96 & 96 & 96 & 88 & 92 & 88 & 92 & 88 & 80 & 76 \\
\hline DP & 2,50 & 2,91 & 2,74 & 3,12 & 3,10 & 4,31 & 3,82 & 5,35 & 3,33 & 2,67 & 4,81 & 2,11 \\
\hline Quartil 1 & 96 & 96 & 96 & 92 & 92 & 88 & 92 & 84 & 92 & 84 & 80 & 76 \\
\hline Tamanho & 17 & 17 & 17 & 17 & 100 & 92 & 96 & 88 & 96 & 88 & 88 & 80 \\
\hline IC & 1,19 & 1,38 & 1,30 & 1,49 & 21 & 21 & 21 & 21 & 9 & 9 & 9 & 9 \\
\hline Valor de $p$ & \multicolumn{2}{|c|}{$0,083 \#$} & \multicolumn{2}{|c|}{$0,052 \#$} & \multicolumn{2}{|c|}{$<0,001^{*}$} & \multicolumn{2}{|c|}{$<0,001^{*}$} & \multicolumn{2}{|c|}{$0,010^{*}$} & \multicolumn{2}{|c|}{$0,040^{*}$} \\
\hline
\end{tabular}

Legenda: $\mathrm{DP}$ = desvio-padrão; IC = intervalo de confiança; NMC = nível máximo de conforto; OD = orelha direita; OE = orelha esquerda

Teste Wilcoxon; * Resultados estatisticamente significantes; \# Resultados próximos ao nível de significância 
tisticamente significante dos valores percentuais de IPRF entre as orelhas.

Nas Tabelas 2 e 3 e Figuras 1, 2 e 3, estão os valores de IPRF aplicados no NMC e a $40 \mathrm{~dB}$ NS nas orelhas direita, esquerda e em ambas, segundo o grau de perda auditiva. Como houve diferença entre as orelhas, foi realizada uma comparação entre o IPRF obtido no NMC e a $40 \mathrm{~dB}$ NS (teste de Wilcoxon) para OD, OE e ambas simultaneamente (denominada de geral).

Na Tabela 2 e Figura 1, observa-se que, para perda auditiva neurossensorial de grau leve, houve diferença significante somente na análise geral.

Na Tabela 3 e Figuras 2 e 3 verifica-se que nas perdas au-

Tabela 2. Medidas descritivas e resultado do teste Wilcoxon para comparação do Índice Percentual de Reconhecimento de Fala obtido nas orelhas direita e esquerda e em ambas as orelhas no NMC e a $40 \mathrm{~dB}$ NS para perda auditiva de grau leve (Grupo I)

\begin{tabular}{|c|c|c|c|c|c|c|}
\hline \multirow{2}{*}{ Medida estatística } & \multicolumn{2}{|c|}{ Orelha direita } & \multicolumn{2}{|c|}{ Orelha esquerda } & \multicolumn{2}{|c|}{ Geral } \\
\hline & NMC & $40 \mathrm{~dB} N \mathrm{NS}$ & NMC & $40 \mathrm{~dB} N S$ & NMC & $40 \mathrm{~dB} N \mathrm{NS}$ \\
\hline Média & 98,12 & 97,18 & 96,71 & 95,53 & 97,41 & 96,35 \\
\hline Mediana & 100 & 96 & 96 & 96 & 96 & 96 \\
\hline DP & 2,50 & 2,74 & 2,91 & 3,12 & 2,76 & 3,01 \\
\hline Quartil 1 & 96 & 96 & 96 & 92 & 96 & 96 \\
\hline Quartil 3 & 100 & 100 & 100 & 96 & 100 & 100 \\
\hline Tamanho & 17 & 17 & 17 & 17 & 34 & 34 \\
\hline IC & 1,19 & 1,30 & 1,38 & 1,49 & 0,93 & 1,01 \\
\hline Valor de $p$ & \multicolumn{2}{|c|}{0,157} & \multicolumn{2}{|c|}{0,166} & \multicolumn{2}{|c|}{$0,050^{*}$} \\
\hline
\end{tabular}

Legenda: $\mathrm{NMC}$ = nível máximo de conforto; $\mathrm{DP}$ = desvio-padrão; IC = intervalo de confiança

Teste Wilcoxon; * Resultados estatisticamente significantes

Tabela 3. Medidas descritivas e resultado do teste Wilcoxon para comparação do Índice Percentual de Reconhecimento de Fala obtido nas orelhas direita e esquerda e em ambas as orelhas no NMC e a $40 \mathrm{~dB}$ NS para perda auditiva de grau moderado (Grupo II) e moderadamente severo (grupo III)

\begin{tabular}{|c|c|c|c|c|c|c|c|c|c|c|c|c|}
\hline \multirow{3}{*}{$\begin{array}{l}\text { Medida } \\
\text { estatística }\end{array}$} & \multicolumn{6}{|c|}{ Moderado (Grupo II) } & \multicolumn{6}{|c|}{ Moderadamente severo (Grupo III) } \\
\hline & \multicolumn{2}{|c|}{ Orelha direita } & \multicolumn{2}{|c|}{ Orelha esquerda } & \multicolumn{2}{|c|}{ Geral } & \multicolumn{2}{|c|}{ Orelha direita } & \multicolumn{2}{|c|}{ Orelha esquerda } & \multicolumn{2}{|c|}{ Geral } \\
\hline & NMC & $40 \mathrm{~dB} N \mathrm{~N}$ & NMC & $40 \mathrm{~dB} N \mathrm{~N}$ & NMC & $40 \mathrm{~dB} N S$ & NMC & $40 \mathrm{~dB} N S$ & NMC & $40 \mathrm{~dB} N S$ & NMC & $40 \mathrm{~dB} N \mathrm{NS}$ \\
\hline Média & 96,00 & 93,14 & 89,90 & 87,62 & 92,95 & 90,38 & 92,89 & 83,11 & 87,11 & 77,78 & 90,00 & 80,44 \\
\hline Mediana & 96 & 92 & 88 & 88 & 92 & 88 & 92 & 80 & 88 & 76 & 88 & 80 \\
\hline $\mathrm{DP}$ & 3,10 & 3,82 & 4,31 & 5,35 & 4,82 & 5,38 & 3,33 & 4,81 & 2,67 & 2,11 & 4,17 & 4,53 \\
\hline Quartil 1 & 92 & 92 & 88 & 84 & 88 & 88 & 92 & 80 & 84 & 76 & 88 & 76 \\
\hline Quartil 3 & 100 & 96 & 92 & 88 & 96 & 96 & 96 & 88 & 88 & 80 & 92 & 80 \\
\hline Tamanho & 21 & 21 & 21 & 21 & 42 & 42 & 9 & 9 & 9 & 9 & 18 & 18 \\
\hline IC & 1,33 & 1,64 & 1,84 & 2,29 & 1,46 & 1,63 & 2,18 & 3,14 & 1,74 & 1,38 & 1,93 & 2,09 \\
\hline Valor de $p$ & \multicolumn{2}{|c|}{$0,001^{*}$} & \multicolumn{2}{|c|}{$0,018^{*}$} & \multicolumn{2}{|c|}{$<0,001^{*}$} & \multicolumn{2}{|c|}{$0,007^{\star}$} & \multicolumn{2}{|c|}{$0,007^{*}$} & \multicolumn{2}{|c|}{$<0,001^{*}$} \\
\hline
\end{tabular}

Legenda: $\mathrm{NMC}$ = nível máximo de conforto; $\mathrm{DP}=$ desvio-padrão; IC = intervalo de confiança

Teste Wilcoxon; * Resultados estatisticamente significantes

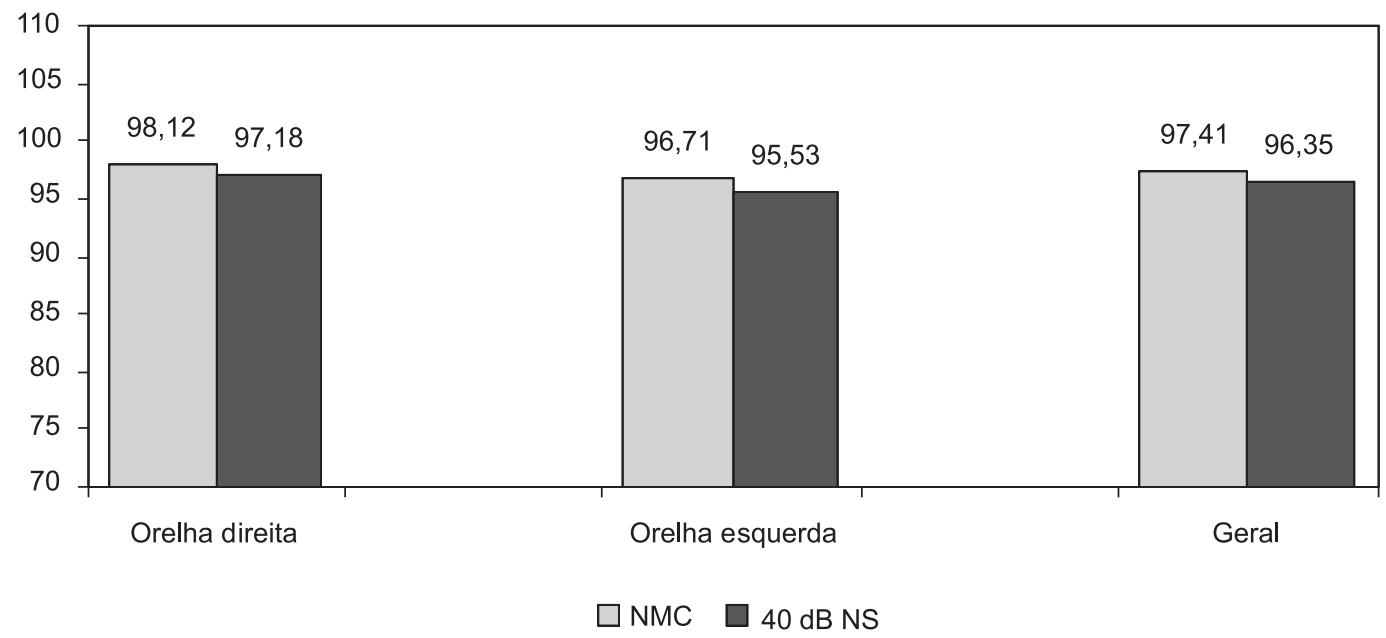

Figura 1. Gráfico descritivo dos valores percentuais do Índice de Reconhecimento de Fala no NMC e a 40 dB NS na orelha direita, esquerda e em ambas, para indivíduos com perda auditiva de grau leve. Legenda: NMC = nível máximo de conforto 


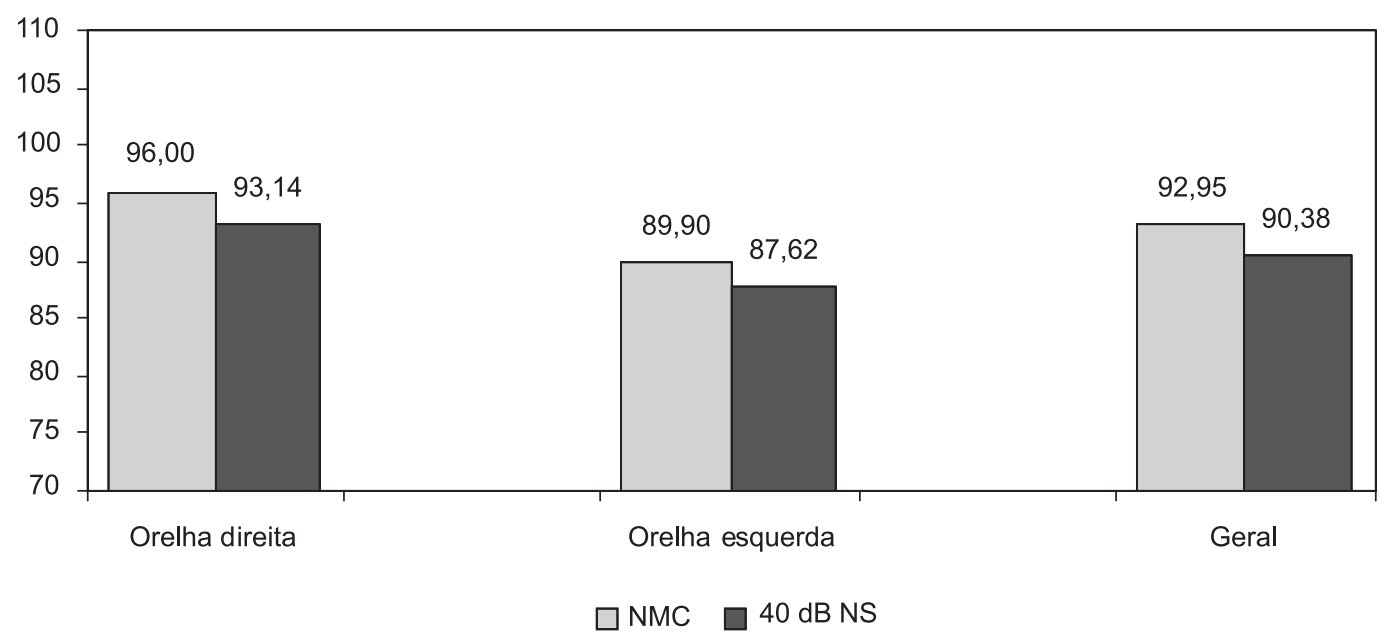

Figura 2. Gráfico descritivo dos valores percentuais do Índice de Reconhecimento de Fala no NMC e a 40 dB NS na orelha direita, esquerda e em ambas para indivíduos com perda auditiva de grau moderado. Legenda: NMC = nível máximo de conforto

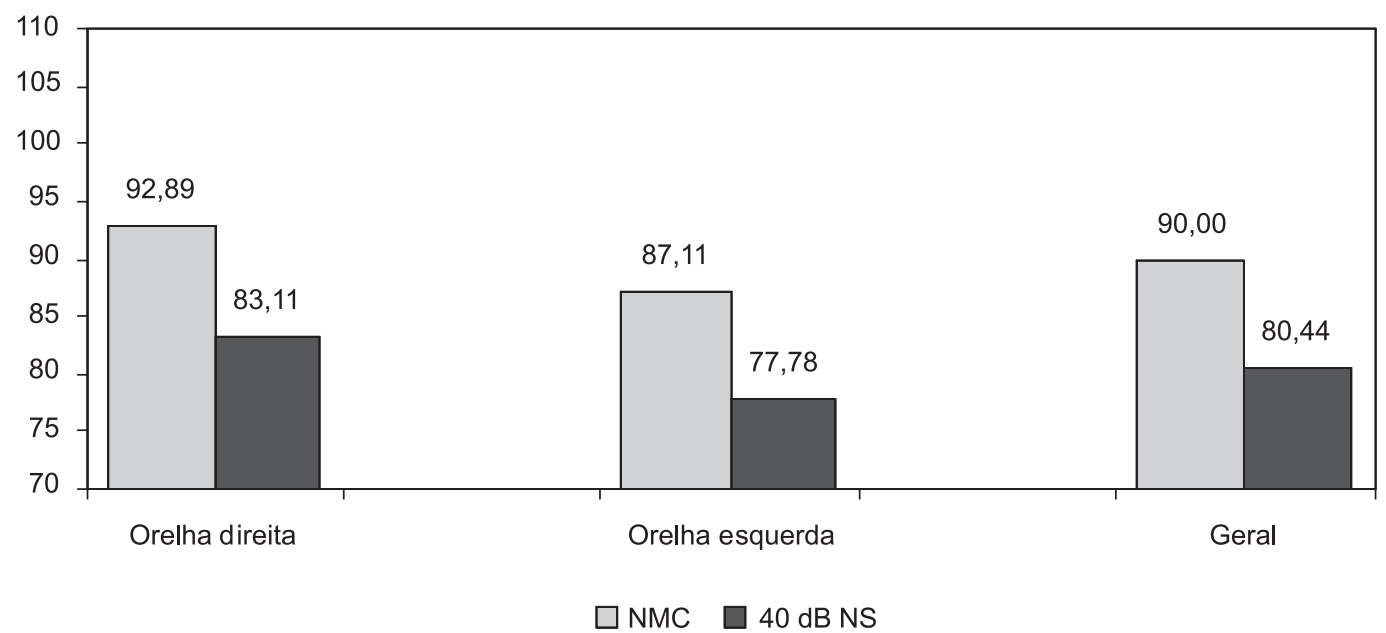

Figura 3. Gráfico descritivo dos valores percentuais do Índice de Reconhecimento de Fala no NMC e a 40 dB NS na orelha direita, esquerda e em ambas para indivíduos com perda auditiva de grau moderadamente severo. Legenda: $\mathrm{NMC}=$ nível máximo de conforto

ditivas neurossensoriais de grau moderado e moderadamente severo, há diferença estatisticamente significante no IPRF obtido no NMC e a $40 \mathrm{~dB}$ NS para ambas as orelhas e no geral.

Para todos os graus de perda (Tabelas 2 e 3 ) pode-se observar que o Índice Percentual de Reconhecimento de Fala é geralmente melhor quando o teste é aplicado no NMC do que a $40 \mathrm{~dB}$ NS.

Foram calculamos os valores médios para a realização do IPRF no nível de máximo conforto para os diferentes graus de perda auditiva e estes valores estão descritos na Figura 4.

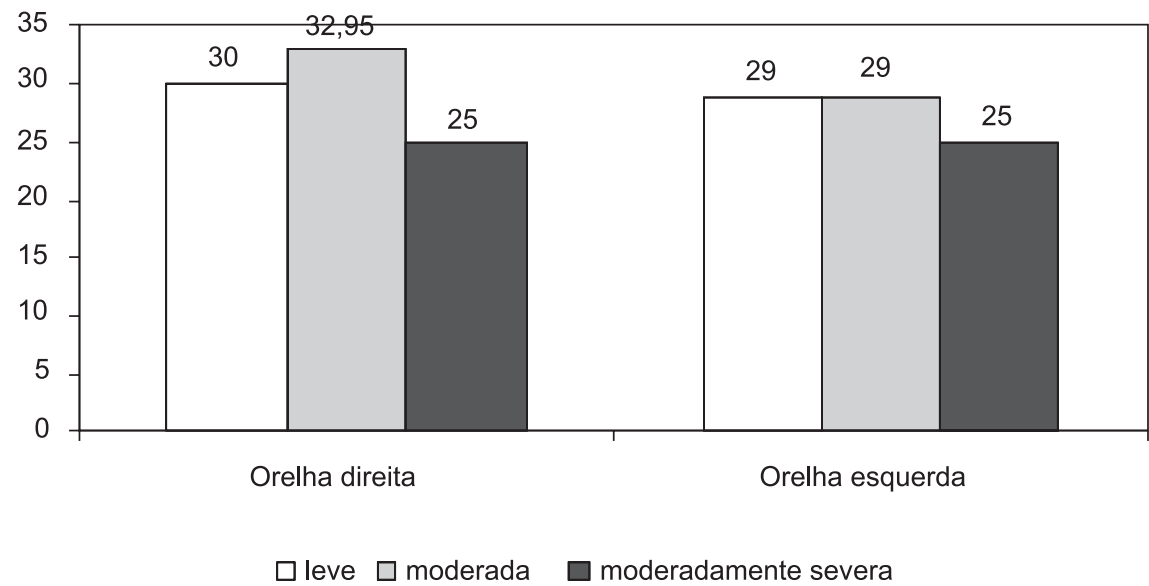

Figura 4. Gráfico descritivo dos valores de nível máximo de conforto (média ponderada) para a realização do Índice Percentual de Reconhecimento de Fala nos três graus perda auditiva 


\section{DISCUSSÃO}

Neste estudo, foram investigadas possíveis diferenças entre o IPRF obtido nas orelhas direita e esquerda no NMC e a 40 dB NS. Não foi encontrado nenhum estudo descritivo sobre o referido assunto.

Durante a aplicação do IPRF, tanto na orelha direita quanto na esquerda ou em ambas, foram encontrados valores maiores para os obtidos no NMC quando comparados aos obtidos a $40 \mathrm{~dB}$ NS.

A redução do Índice de Reconhecimento de Fala, típica dos portadores de deficiência auditiva neurossensorial bilateral pode ser decorrente da privação auditiva dos anos de deficiência ${ }^{(15)}$.

A maioria dos autores recomenda a realização do Índice de Reconhecimento de Fala de 25 a 40 dB NS ${ }^{(16)}$. Porém, não é possível precisar um nível fixo, para determinar o reconhecimento de fala ou os limiares de tom puro, para ser utilizado na avaliação de todos os tipos e graus de perda auditiva ${ }^{(17)}$.

Uma pesquisa ${ }^{(12)}$ sobre o assunto investigou a faixa de intensidades que produz a inteligibilidade de fala máxima para indivíduos com perda auditiva. Foi recomendado que o índice de reconhecimento de fala fosse obtido em pelo menos dois níveis. Os autores do estudo relataram também que os resultados máximos são mais facilmente encontrados no nível mais confortável. Outros autores ${ }^{(10)}$ afirmaram que os valores de reconhecimento de fala dependem também do nível de apresentação, porém o maior nível de apresentação não garante melhor reconhecimento.

Entretanto, para indivíduos apresentando perda auditiva neurossensorial, o desempenho pode estar comprometido quando o estímulo for apresentado em níveis de audição elevados. Assim, acredita-se que o nível confortável para a realização do IPRF esteja em torno de $30 \mathrm{~dB}$ NS.

O princípio do teste é dar ao paciente energia suficiente para que ele ouça todos os fonemas de cada palavra, pois o que se pretende é avaliar o reconhecimento das palavras. Pacientes portadores de perda auditiva neurossensorial podem apresentar diversos graus de comprometimento do reconhecimento de fala, uma vez que há dificuldade de identificar todos os sons mesmo quando as palavras são apresentadas no nível recomendado, ou seja, de 30 a $40 \mathrm{~dB}$ NS. ${ }^{(17)}$
Além disso, em pacientes com perda auditiva neurossensorial, deve-se considerar a possibilidade do fenômeno do recrutamento, pois seu nível de maior conforto encontra-se abaixo do nível de apresentação recomendado para a realização do IPRF (40 dB NS), geralmente em torno de 20 a $25 \mathrm{~dB}$ acima da média dos limiares nas freqüências de fala $(20 \text { a } 25 \mathrm{~dB} \mathrm{NS})^{(17)}$. O que se observa nestes casos é uma piora acentuada dos resultados com aumentos sucessivos da intensidade a partir deste nível. Os autores sugerem que tais níveis geralmente encontram-se em torno de 20 a $25 \mathrm{~dB} \mathrm{NS}^{(18)}$.

O recrutamento é o crescimento anormalmente rápido da sensação de intensidade conforme a intensidade física do som aumenta, causado por lesão das células ciliadas externas (CCE). Dessa forma, infere-se que a maioria desses pacientes eram portadores de recrutamento. A fisiologia coclear explica essa alteração e justifica a realização e os achados do estudo.

Vale ressaltar que os valores encontrados no presente estudo são valores médios, sendo de suma importância, nos casos de perda auditiva neurossensorial, a pesquisa cuidadosa do nível mais confortável para garantir a realização do teste da maneira mais favorável possível, uma vez que o reconhecimento de fala nesses indivíduos ${ }^{(19)}$, já se encontra comprometida.

Ressalta-se, ainda, que os pacientes avaliados eram portadores de perda auditiva neurossensorial de grau leve a moderadamente severo (até $60 \mathrm{~dB}$ ). O IPRF médio variou de $77 \%$ a 98\%.

\section{CONCLUSÕES}

Os resultados obtidos no estudo do Índice Percentual de Reconhecimento de Fala em adultos com perda auditiva neurossensorial possibilitaram concluir que:

Nas perdas auditivas de grau moderado e moderadamente severo, o IPRF obtido no NMC foi melhor do que o obtido a $40 \mathrm{~dB}$ NS.

O IPRF foi melhor na orelha direita.

\section{AGRADECIMENTOS}

À Fundação de Amparo à Pesquisa do Estado de São Paulo (FAPESP), pelo apoio concedido para realização dessa pesquisa.

\begin{abstract}
Purpose: To obtain the Percentage Index of Speech Recognition (PISR) at maximum comfortable level (MCL) in adults with mild to moderately severe (up to $60 \mathrm{~dB}$ ) sensorineural hearing loss. Methods: The subjects evaluated were grouped according to the degree of hearing loss (Groups I, II and III). The groups were further divided into subgroups (IA,IB, IIA, IIB, IIIA, IIIB) as it follows. In the subgroup A of each group, the PISR was determined at $40 \mathrm{~dB}$ SL, beginning with the right ear. The patient was then requested to inform how comfortable the sound was at this level of stimuli presentation, according to a scale with four possibilities: low, comfortable, high and excessively high. After that, the maximum comfortable level (MCL) was obtained, and the PISR was carried out using that intensity level. In the subgroup B of each group, the same procedure was carried out, only reversely, performing initially the PISR at MCL, and then at $40 \mathrm{~dB}$ SL. In this subgroup the test began with the left ear. Results: After evaluating the subjects of the three groups, the mean MCL was calculated. It varied from 25 to $32.95 \mathrm{~dB}$ SL. The subjects had better results in the PISR when stimuli were presented at MCL. Conclusions: PISR evaluation at MCL, for individuals with mild to moderately severe sensorineural hearing loss, provides better Speech Recognition results.
\end{abstract}

Keywords: Hearing loss, sensorineural; Auditory threshold; Audiometry, speech; Speech perception 


\section{REFERÊNCIAS}

1. Gonçalves MS, Tochetto TM. Hiperacusia: uma abordagem teórica. Rev CEFAC. 2005;7(2):234-40.

2. de Miranda EC, Costa MJ. Reconhecimento de sentenças no silêncio e no ruído de indivíduos jovens adultos normo-ouvintes em campo livre. Fono Atual. 2006;8(35):4-12.

3. Freitas CD, Costa MJ. Variabilidade dos limiares de reconhecimento de fala no teste-reteste de indivíduos normo-ouvintes. Fono Atual. 2006;8(35):30-40.

4. Longone E, Borges AC. Teste de reconhecimento de fala em indivíduos portadores de perda auditiva neurossensorial. Pró-Fono. 1997;9(1):3-9.

5. Matos AT, Magalhães MV, Gómez SG. Índice de reconhecimento de fala na presbiacusia. Arq Int Otorrinolaringol [internet] 2007 [citado 2009 Out 22]; 11 (2). Disponível em: http://www.arquivosdeorl.org.br/ conteudo/acervo_port.asp?id=420

6. Costa LP, Iorio MC. Próteses auditivas: avaliações objetivas e subjetivas em usuários de amplificação linear e não-linear. Pró-Fono. 2006;18(1):21-30.

7. Barros PF, Queiroga BA. As dificuldades encontradas no processo de adaptação de aparelho de amplificação sonora individual em indivíduos Idosos. Rev CEFAC. 2006;8(3):375-85.

8. Silva RC, Bevilacqua MC, Mitre EI, Moret AL, Teste de Percepção de Fala para palavras dissílabas. Rev CEFAC. 2004;6(2):209-14.

9. Batista FP, Rezende GM, Vaz RN, Mitre EI. Análise comparativa do índice de reconhecimento de fala (IRF) em pacientes presbiacúsicos com e sem recrutamento. Rev CEFAC. 2006;8(2):230-4.

10. Lopes Filho O, organizador. Tratado de fonoaudiologia. São Paulo: Roca;1997.
11. Goffi-Gomes MV, Pedalini ME. Testes audiológicos para identificação de alterações retrococleares. In: Lopes Filho O, organizador. Tratado de Fonoaudiologia. São Paulo: Ed. Roca; 1997. p.127-47.

12. Katz J. Tratado de audiologia clínica. 4a ed. São Paulo: Manole; 1999.

13. Chaves AD, Nepomuceno LA, Rossi AG, Mota HB, Pillon L. Reconhecimento de fala: uma descrição de resultados obtidos em função do número de sílabas dos estímulos. Pró-Fono. 1999;11(1):53-8.

14. Mangabeira PL, Pen M. Elementos de psicoacústica. In: Munhoz M, Caovilla HH, Silva MLG, Ganança MM. Audiologia clínica. São Paulo: Atheneu, 2000. p. 11-18.

15. Prates LP, Iorio MC. Aclimatização: estudo do reconhecimento de fala em usuários de próteses auditivas. Pró-Fono. 2006;18(3):259-66.

16. Wilson RH, Strouse AL. Audiometria com estímulos de fala. In: Musiek FE, Rintelmann NF. Perspectivas atuais em avaliação auditiva. São Paulo: Manole, 2001. p. 21-54.

17. Beattie RC, Zipp JA. Range of intensities yelding PB Max and the threshold for monosyllabic words for hearing-impaired subjects. J Speech Hear Disord. 1990;55(3):417-26.

18. Almeida LC. Índice percentual de reconhecimento de fala em indivíduos com perda auditiva neurossensorial [tese]. São Paulo (SP): Universidade Federal de São Paulo - Departamento de Fonoaudiologia; 2003.

19. Caporali SA, Silva JA. Reconhecimento de fala no ruído em jovens e idosos com perda auditiva. Rev Bras Otorrinolaringol. 2004;70(4):52532. 